OAI-PMH: http://www.indteca.com/ojs/index.php/Revista Scientific/oai

Artículo Original / Original Article

\title{
Procrastinación e incremento del estrés en docentes y estudiantes universitarios frente a la educación online
}

\author{
Autor: César Raúl Méndez Carpio \\ Universidad Católica de Cuenca, UCACUE \\ cmendezc@ucacue.edu.ec \\ Cuenca, Ecuador \\ https://orcid.org/0000-0003-0582-0107
}

\section{Resumen}

La incidencia de la pandemia en el mundo ha dado un giro exponencial a los procesos sociales $y$, por ende, a la modalidad de formación en los procedimientos académicos de todas las instituciones educativas. Con ello y en concordancia con el análisis de la procrastinación y su incidencia en el incremento del estrés tanto en estudiantes como en docentes universitarios, que han tenido que adaptarse a la enseñanza online que, se ha propuesto como objetivo, la necesidad de observar estas conductas mediante la aplicación metodológica que consideró el enfoque mixto cuali-cuantitativo, apoyado en un análisis descriptivo. Ha sido necesario, revisar y priorizar presupuestos teóricos de varios documentos publicados; además, se aplicó un cuestionario estructurado a una muestra intencional lo que ha permitido obtener como resultados, el análisis de conductas de procrastinación y características propias del estrés que se han visto incrementadas en la muestra estudiada tales como: emociones, pensamientos, conductas y cambios físicos los cuales coadyuvan a proponer el uso adecuado del tiempo en todas las actividades académicas y sociales, para facilitar la consecución de las tareas cotidianas, plantear alternativas de solución minimizando el impacto y desarrollando actitudes de apoyo a la gestión docente y discente de los universitarios.

Palabras clave: procrastinación; estrés; enseñanza online; confinamiento social.

Código de clasificación internacional: 3201.05 - Psicología clínica.

Cómo citar este artículo:

Méndez, C. (2021). Procrastinación e incremento del estrés en docentes y estudiantes universitarios frente a la educación online. Revista Scientific, 6(20), 62-78, e-ISSN: 2542-2987. Recuperado de: https://doi.org/10.29394/Scientific.issn.2542-2987.2021.6.20.3.62-78

Fecha de Recepción: 09-01-2021
Fecha de Aceptación: 19-03-2021
Fecha de Publicación: 05-05-2021 
OAI-PMH: http://www.indteca.com/ojs/index.php/Revista Scientific/oai

Artículo Original / Original Article

\title{
Procrastination and increased stress in teachers and university students in the face of online education
}

\begin{abstract}
The incidence of the pandemic in the world has given an exponential turn to social processes and, therefore, to the modality of training in the academic procedures of all educational institutions. With this and in accordance with the analysis of procrastination and its incidence in the increase of stress in both students and university teachers, who have had to adapt to online teaching, which has been proposed as an objective, the need to observe these behaviors Through the methodological application that considered the mixed quali-quantitative approach, supported by a descriptive analysis. It has been necessary to review and prioritize theoretical budgets of various published documents; In addition, a structured questionnaire was applied to an intentional sample, which has allowed to obtain as results, the analysis of procrastination behaviors and characteristics of stress that have been increased in the studied sample, such as: emotions, thoughts, behaviors and physical changes which help to propose the appropriate use of time in all academic and social activities, to facilitate the achievement of daily tasks, propose alternative solutions minimizing the impact and developing attitudes of support to the teaching and learning management of university students.
\end{abstract}

Keywords: procrastination; stress; online teaching; social confinement. International classification code: 3201.05 - Clinical psychology.

\footnotetext{
How to cite this article:

Méndez, C. (2021). Procrastination and increased stress in teachers and university students in the face of online education. Revista Scientific, 6(20), 62-78, e-ISSN: 2542-2987. Recovered from: https://doi.org/10.29394/Scientific.issn.2542-2987.2021.6.20.3.62-78
}

Date Received: 09-01-2021
Date Acceptance: 19-03-2021
Date Publication: 05-05-2021 


\section{Introducción}

A lo largo de la historia, la humanidad ha experimentado muchas situaciones problemáticas que han servido de motivación para su proceso de evolución constante en todas las ramas del saber científico. La Educación ha sido uno de los campos de acción del conocimiento que ha sufrido un progreso más evidente a la adaptación del avance técnico, tecnológico, industrial, entre otros, y que su cambio continuo ha denotado como muy notorio para el bien común de la sociedad actual. En concordancia con Cueva, García y Martínez (2019):

La concepción de las sociedades desarrolladas con alta demanda en el uso de las Tecnologías de la Información y la Comunicación (TIC) que hoy se divisa, es producto del acelerado desarrollo y explosión de la evolución de la ciencia; la que está sustentada en las ansias del saber, conocer, dominar y buscar mejores resultados en los procesos productivos y sociales del hombre a lo largo de la historia [...] (pág. 207).

Ahora bien, toda transformación trae consigo ventajas y desventajas; y en este contexto, precisamente en el año 2020, en el que, por motivo de la pandemia mundial provocada por la COVID-19 y el confinamiento social, han ocasionado el colapso de muchos, por no decir todos, los sistemas sanitarios, y han impactado los procedimientos educativos a nivel mundial. La pandemia en mención ha conseguido modificar todos los procedimientos de la vida diaria del ser humano y parte fundamental de ellos; la educación presencial, la misma que ha tenido que adaptarse de forma drástica e inmediata para convertirse en una educación virtual u online, lo que resulta inquietante tanto para docentes como estudiantes en todos los niveles académicos $y$, en especial, a los universitarios que asiste el presente estudio.

Este cambio pedagógico ha ocasionado múltiples variaciones en la conducta y la actuación de docentes y estudiantes, que inciden en el 
incremento del estrés, el mismo que, según la definición de Espinosa-Castro, Hernández-Lalinde, Rodríguez, Chacín y Bermúdez-Pirela (2020): "puede ser definido como el proceso que se inicia ante un conjunto de demandas ambientales que recibe el individuo, a los cuáles debe dar una respuesta adecuada, poniendo en marcha sus recursos de afrontamiento" (pág. 64); y que podría ser reflejo de una de las causas notorias como la obligatoriedad en la postergación de actividades académicas, y muchas personales que a la larga tendrían repercusiones en su vida futura.

El estrés está inevitablemente formando parte de nuestro entorno e incide en la salud mental de la población estudiantil. Como afirman Suárez y Díaz (2015): "[...] el estrés académico es un elemento que favorece el estrés crónico y el deterioro de la salud mental" (pág. 302): por tal razón, debemos centrar la atención en éste como uno de los ámbitos donde más tiempo ocupamos nuestra vida, y darle relevancia a la problemática citada en concordancia con lo que señala Silva-Ramos, López-Cocotle y Meza-Zamora (2020):

El estrés está presente en todos los ámbitos de acción de un individuo, incluido el educativo, de ahí la preocupación de los integrantes del equipo de salud para conocer las implicaciones que este tiene sobre el rendimiento de los estudiantes; es decir, el estrés que los estudiantes experimentan durante su tránsito escolar (pág. 76).

Una de las variables que inciden en el incremento del estrés es la procrastinación, concepto que definen Atalaya y García (2019a): como "posponer actividades en su desarrollo y culminación por preferir otras tareas de interés, y el tiempo es utilizado para socializar, priorizar la recreación o simplemente no hacer tareas" (pág. 374). En concordancia con los autores, es necesario considerar el uso adecuado del tiempo con el fin de evitar o minimizar el impacto que ello significa en el aumento del estrés notado.

El uso adecuado del tiempo, en estos momentos de aislamiento social, 
conlleva tener contacto social mínimo con otras personas para minimizar el riesgo y evitar consecuencias que causen problemas negativos que, por la situación, afecten el desarrollo normal de actividades y, en especial, las educativas, motivo de este estudio, considerando que la mayoría de estudiantes se encuentran en contacto con la tecnología a través del uso de la internet, las plataformas de videojuegos, la televisión, teléfonos móviles, utilizados para tiempos extensos de ocio.

Es importante considerar el tiempo invertido en ello, la adecuada distribución de este, ya que como indican Moncada y Chacón (2012): el uso inadecuado y excesivo de la tecnología sin control de tiempo, traerá efectos tanto psicológicos como fisiológicos positivos o negativos en el aprendizaje, su desarrollo y aplicación en la puesta en práctica al enfrentar nuevas situaciones.

En referencia al personal docente y en este contexto, Gené-Badia, RuizSánchez, Obiols-Masó, Oliveras y Lagarda (2016): aseveran que la población adulta es vulnerable a padecer estados de soledad, apoyados estos del sedentarismo y fatiga que, cuando se unen fuertemente, conllevan a producir enfermedades de tipo cardiovascular, enfermedades de vías respiratorias continuas, entre otras. A pesar de lo que se menciona, Barba (2019): sugiere que se deben analizar varios aspectos positivos tales como; el fortalecimiento de los lazos afectivos familiares, mejoras en la adaptación al lugar y entorno en el que se desarrollan permitiéndoles actuar y reaccionar de mejor manera al proceso del confinamiento social que produce los cambios.

Durante el año 2020, la pandemia por el virus COVID-19, ha forzado a todas las personas a tomar medidas para salvaguardar su bienestar. Los gobiernos a nivel mundial declararon como primera medida el confinamiento social en los hogares, lo que conllevó al deterioro de la salud física producto del sedentarismo. El paso drástico a la modalidad de estudio virtual ha sido un detonante tanto para los estudiantes como para los docentes en su desarrollo y aplicación emergente en los procesos de formación que, unido a todo lo 
analizado, ha incrementado conductas de procrastinación y, por ende, los niveles de estrés en este sector importante de la población mundial. En referencia a la educación virtual, Nieto (2012), manifiesta que:

$\mathrm{Si}$, por otro lado, la "educación" es la acción y el efecto de educar, o el modo en que se ha educado una persona, ello nos lleva a la arena donde se enfrentan lo "real" con lo "virtual", la "realidad" con la "virtualidad". De donde la virtualidad será, entonces, el eclecticismo de lo humano: lo real, lo simbólico y lo imaginario (pág. 140).

En este contexto, se hace apremiante plantear y responder la siguiente interrogante: ¿Cuál es la incidencia de la procrastinación en el incremento de los niveles de estrés de docentes y estudiantes universitarios frente al paso emergente a la educación online?.

El hombre como ser humano, es propenso al padecimiento de varios trastornos tanto físicos como mentales producto del ritmo de vida que le ha tocado llevar actualmente para adaptarse a cambios vertiginosos como son la globalización y sus múltiples factores tales como: adaptación al cambio social, cambio tecnológico, situaciones y experiencias de cumplimiento de tareas diarias en los diferentes escenarios en el que se desenvuelven con relación a su función y actividad. Una de las afecciones más frecuentes y consideradas por muchos hoy como situación normal, es el estrés, que está presente en todos los individuos y, en especial, en la población motivo de estudio, los docentes y estudiantes universitarios en su relación con el proceso de enseñanza aprendizaje en línea.

Al conocer la incidencia que tiene en el aumento del estrés la postergación de actividades académicas y de índole personal en docentes y estudiantes universitarios frente a la educación online, es preciso considerar la conciencia social inmersa en los cambios de comportamiento que deben ser analizados al momento de participar en la modalidad virtual. La educación en el nivel superior no puede estar inmerso dentro de un ambiente estresado 
puesto que puede disminuir su propósito y convertirse en una educación mediocre con múltiples vacíos educativos y, producto de ello, en la desidia tanto en la enseñanza por parte del docente como el aprendizaje por parte de los estudiantes.

La importancia de este proceso investigativo, radica en determinar el uso adecuado del tiempo, y presentar alternativas que sean consideradas para evitar la procrastinación y disminuir los niveles de estrés en la gestión académica de los involucrados, tomar consciencia de ello y conseguir un mejor orden social en todos los niveles en el que incide esta práctica. Así pues, se pretende incrementar la importancia que tiene la educación virtual actual, las implicaciones de responsabilidad que se generan tanto en docentes como en estudiantes evitando de esta manera, posponer las principales obligaciones académicas en contra del aumento del estrés que se pueda generar.

La investigación pretende entonces analizar conductas de procrastinación y su incidencia en los niveles de estrés en docentes y estudiantes universitarios que utilizan la educación online, con el fin de proponer actividades académicas y sociales que conlleven al uso adecuado del tiempo y minimizan el impacto.

Es necesario además, reforzar la interrogante de investigación planteada a través del análisis documental y referencial teórico sobre las variables de estudio, la recolección de información de primera línea aprovechando el acercamiento directo tanto con docentes y estudiantes de nivel superior para que los datos obtenidos sean lo suficientemente fiables y fruto de la experiencia diaria en este paso acelerado no planificado a la enseñanza online, y plantear, a través de una propuesta, tareas y oportunidades de mejora enfocadas a mitigar el problema del estrés, minimizar el impacto de la procrastinación y con ello considerar el uso adecuado del tiempo en los distintos procesos académicos y sociales de docentes y estudiantes del sector de educación en mención. 


\section{Metodología (Materiales y métodos)}

Para el proceso metodológico de la presente investigación, se consideró el enfoque mixto; cuali-cualitativo de tipo descriptivo, puesto que el investigador comenzó sondeando el mundo social en el que se desenvuelve el problema, apoyado en la técnica de revisión bibliográfica basada en la información publicada en bases de datos científicas y revistas indexadas sobre las variables de estudio. Así mismo, se aplicó una encuesta estructurada utilizando formularios en línea con apoyo de la herramienta de Google drive a estudiantes y docentes universitarios considerando una muestra intencional de 354 individuos considerados en las categorías de análisis; perteneciendo el $11,9 \%$ a docentes y el $88,1 \%$ a estudiantes universitarios, verificando así los indicadores y detonantes del incremento del estrés producto de la procrastinación.

La aplicación del estudio permitió además analizar el uso adecuado del tiempo con enfoque en la vida académica, la importancia de clasificar lo importante y necesario de la gestión docente o discente y promover una cultura de conciencia del estrés y sus consecuencias futuras en la salud de las personas ya que cada vez el mundo social se vuelve trágico y vulnerable ante el manejo de emociones, pensamientos, conductas y cambios físico de acuerdo con las circunstancias.

\section{Resultados (análisis e interpretación de los resultados)}

El estudio consiguió analizar el grado de incidencia de las conductas de procrastinación en los niveles de estrés en docentes y estudiantes universitarios frente al paso emergente a la educación online verificando que muchas de las veces, se las pasa por alto y no se mide el riesgo para poderlo a tiempo mitigar el impacto, considerar el uso adecuado del tiempo tanto en tareas académicas como sociales de los individuos. Como afirman Atalaya y García (2019b): "el término procrastinación se ha convertido en un fenómeno 
típico en nuestra sociedad, cuya actividad de posponer tareas es un acto irresponsable del sujeto (pág. 364)".

Al aplicarse la encuesta, se desarrollaron dimensiones de estudio que permitieron triangular la investigación en sus diferentes enfoques tales como: análisis de antecedentes de las características de procrastinación, incidencia en el aumento de estrés verificando cambios de actitudes en lo referente a emociones, pensamientos, conductas e inclusive cambios manifestados en la salud física de los integrantes de la muestra señalada. En relación con las dimensiones comentadas para conseguir el propósito de estudio y sus categorías, se expresan de forma porcentual los resultados en la tabla 1.

Tabla 1. Resultados de la encuesta para analizar conductas de procrastinación y su incidencia en los niveles de estrés.

\begin{tabular}{|c|c|c|}
\hline ANTECEDENTES DE PROCRASTINACIÓN & SI & NO \\
\hline $\begin{array}{l}\text { ¿Considera que el paso urgente a la modalidad de educación virtual fue } \\
\text { positiva? }\end{array}$ & $51,1 \%$ & $48,9 \%$ \\
\hline $\begin{array}{l}\text { ¿Durante la pandemia ocurrida por la presencia de COVID-19 ha tenido } \\
\text { que dejar de hacer actividades urgentes? }\end{array}$ & $65,0 \%$ & $35,0 \%$ \\
\hline $\begin{array}{l}\text { ¿Le preocupa a Usted el cumplimiento de tareas y obligaciones en los } \\
\text { tiempos planificados por la institución educativa? }\end{array}$ & $88,4 \%$ & $11,6 \%$ \\
\hline $\begin{array}{l}\text { ¿Considera Ud. que su nivel de estrés aumenta al postergar la elaboración } \\
\text { de actividades urgentes? }\end{array}$ & $89,0 \%$ & $11,0 \%$ \\
\hline $\begin{array}{l}\text { ¿Considera que la educación virtual actual ha provocado que se postergan } \\
\text { actividades importantes? }\end{array}$ & $77,4 \%$ & $22,6 \%$ \\
\hline $\begin{array}{l}\text { ¿Cree que la educación virtual actual requiere que se le dedique más } \\
\text { tiempo que en la modalidad presencial? }\end{array}$ & $67,8 \%$ & $32,2 \%$ \\
\hline $\begin{array}{l}\text { ¿Está de acuerdo con los tipos de prácticas educativas actuales tales } \\
\text { como tareas deberes proyectos etc., en la modalidad virtual aplicada por } \\
\text { su institución? }\end{array}$ & $63,8 \%$ & $36,2 \%$ \\
\hline $\begin{array}{l}\text { ¿Considera que se aplican herramientas tecnológicas adecuadas } \\
\text { analizando el uso adecuado del tiempo de los estudiantes? }\end{array}$ & $76,8 \%$ & $23,2 \%$ \\
\hline $\begin{array}{l}\text { ¿Cree Ud. que planifica adecuadamente su tiempo vs sus actividades } \\
\text { personales, educativas y otras? }\end{array}$ & $56,8 \%$ & $43,2 \%$ \\
\hline $\begin{array}{l}\text { ¿Cree Ud. que la institución educativa de la cual forma parte, considera los } \\
\text { tiempos académicos y es consciente de su tiempo personal? }\end{array}$ & $76,8 \%$ & $23,2 \%$ \\
\hline ANÁLISIS DE EMOCIONES & \multirow[b]{2}{*}{ SI } & \multirow[b]{2}{*}{ NO } \\
\hline $\begin{array}{l}\text { Considera Ud. que en los últimos tiempos ha sentido el aumento de las } \\
\text { siguientes emociones: }\end{array}$ & & \\
\hline $\begin{array}{ll} & \text { Ansiedad }\end{array}$ & $70,1 \%$ & $29,9 \%$ \\
\hline Miedo & $29,4 \%$ & $70,6 \%$ \\
\hline
\end{tabular}


Instituto Internacional de Investigación y Desarrollo Tecnológico Educativo INDTEC, C.A.

DOI: https://doi.org/10.29394/Scientific.issn.2542-2987.2021.6.20.3.62-78

OAI-PMH: http://www.indteca.com/ojs/index.php/Revista Scientific/oai

Artículo Original / Original Article

\begin{tabular}{|c|c|c|}
\hline Irritabilidad & $56,5 \%$ & $43,5 \%$ \\
\hline Confusión & $40,1 \%$ & $59,9 \%$ \\
\hline ANÁLISIS DE PENSAMIENTOS & \multirow[b]{2}{*}{ SI } & \multirow[b]{2}{*}{ NO } \\
\hline $\begin{array}{l}\text { Considera Ud. que en los últimos tiempos ha sentido el aumento de las } \\
\text { siguientes Pensamientos: }\end{array}$ & & \\
\hline Dificultad para concentrarse & $64,1 \%$ & $35,9 \%$ \\
\hline pensamientos repetitivos & $30,5 \%$ & $69,5 \%$ \\
\hline excesiva autocrítica & $26,6 \%$ & $73,4 \%$ \\
\hline olvidos & $42,1 \%$ & $57,9 \%$ \\
\hline preocupación por el futuro & $59,6 \%$ & $40,4 \%$ \\
\hline ANÁLISIS DE CONDUCTAS & \multirow[b]{2}{*}{ SI } & \multirow[b]{2}{*}{ NO } \\
\hline $\begin{array}{l}\text { Considera Ud. que en los últimos tiempos ha sentido el aumento de las } \\
\text { siguientes Conductas: }\end{array}$ & & \\
\hline Dificultades en el habla & $30,8 \%$ & $69,2 \%$ \\
\hline Risa nerviosa & $33,3 \%$ & $66,7 \%$ \\
\hline Trato brusco en las relaciones sociales & $30,8 \%$ & $69,2 \%$ \\
\hline Llanto & $46,3 \%$ & $53,7 \%$ \\
\hline Apretar las mandíbulas & $26,0 \%$ & $74,0 \%$ \\
\hline Aumento del consumo de tabaco & $5,4 \%$ & $94,6 \%$ \\
\hline Alcohol & $3,1 \%$ & $96,9 \%$ \\
\hline ANÁLISIS DE CAMBIOS FÍSICOS & \multirow[b]{2}{*}{ SI } & \multirow[b]{2}{*}{ NO } \\
\hline $\begin{array}{l}\text { Considera Ud. que en los últimos tiempos ha sentido el aumento de los } \\
\text { siguientes Cambios físicos: }\end{array}$ & & \\
\hline Músculos contraídos & $26,0 \%$ & $74,0 \%$ \\
\hline Dolor de Cabeza & $68,4 \%$ & $31,6 \%$ \\
\hline Problemas de espalda o cuello & $70,6 \%$ & $29,4 \%$ \\
\hline Malestar estomacal & $30,8 \%$ & $69,2 \%$ \\
\hline Fatiga & $47,5 \%$ & $52,5 \%$ \\
\hline Infecciones & $5,6 \%$ & $94,4 \%$ \\
\hline Palpitaciones y respiración agitada & $23,2 \%$ & $76,8 \%$ \\
\hline
\end{tabular}

Fuente: El Autor (2021).

Se puede anotar con referencia a los antecedentes de procrastinación, que más de la mitad de los docentes y estudiantes encuestados; el $51,1 \%$ califican como positivo al paso a la modalidad virtual en contra de un $48,9 \%$ que creen no serlo. Reconocen además el 65,0\% haber dejado de hacer actividades urgentes durante la pandemia ocurrida por la presencia de la 
COVID-19 y el 35,0\% indican no haber actuado de esta manera. Al 88,4\% les preocupa el cumplimiento de tareas y obligaciones en los tiempos planificados, frente a un $11,6 \%$ que dice no preocuparlos.

En relación con el aumento del estrés frente a la postergación de actividades está de acuerdo el $89,0 \%$ de los encuestados frente al 11,0\% que afirma no mutarse. Motivo importante notado por el $77,4 \%$ es considerar que la educación virtual ha provocado que se posterguen actividades importantes, ante lo cual el 22,6\% está en desacuerdo. Así mismo con relación a manifestar que la educación virtual actual requiere que se le dedique más tiempo que en la modalidad presencial, están de acuerdo el $67,8 \%$ frente al $32,2 \%$ señala que no.

Ya en el campo pedagógico al preguntar si está de acuerdo con los tipos de prácticas educativas actuales tales como tareas deberes proyectos etc., en la modalidad virtual aplicada por su institución, afirman estarlo el 63,8\% mientras que el $36,2 \%$ dicen que no. Consecuencia de lo notado, es considerado por el $76,8 \%$, que se aplican herramientas tecnológicas adecuadas frente al uso adecuado del tiempo de los estudiantes y el 23,2\% consideran que no se lo hace. Así mismo al preguntar si se planifica adecuadamente el tiempo vs la realización de las actividades personales, educativas y otras, contestan que sí el $56,8 \%$ frente a una negación del 43,2\% de la muestra encuestada que se corrobora con analizar si la institución educativa de la cual forma parte considera los tiempos académicos y es consciente de su tiempo personal, afirman que sí el $76,8 \%$ y que no, el $23,2 \%$.

Es evidente notar en el campo de las emociones el incremento demostrado como detonante del aumento del estrés, reconociendo el $70,1 \%$ de la población de estudio haber tenido aumento de ansiedad, el 29,9\% complementario dice que no lo ha notado, el $29,4 \%$ dice tener miedo frente al $70,6 \%$ que afirma no haberlo sentido, el $56,5 \%$ ha sentido irritabilidad, en contra del $43,5 \%$ que no lo ha hecho y por último en este espacio de las 


\section{Artículo Original / Original Article}

emociones, el $40,1 \%$ afirma tener confusión, frente al $59,9 \%$ que no lo ha tenido.

Se desarrollaron criterios que tienen relación con la modificación de pensamientos que generan estrés, siendo notorio en el $64,1 \%$ el aumento en la dificultad para concentrarse, en relación con el 35,9\% que no lo ha hecho; el $30,5 \%$ señala haber tenido pensamientos repetitivos y el $69,5 \%$ de los mismos no lo ha considerado; lo que conlleva también a tener pensamientos autocríticos en un $26,6 \%$ y un $73,4 \%$ indican no haberlos tenido; por otro lado, afirman haber aumentado las situaciones de olvido en un $42,1 \%$ y el $57,9 \%$ restante no ha demostrado tener los mismos; concomitante a ello se ha demostrado preocupación por el futuro en un 59,6\% de los encuestados siendo contrario a ellos el $40,4 \%$.

Por otro lado, en un análisis de conductas se expresa el haber presentado dificultades en el habla en el 30,8\% de la población, mientras que el $69,2 \%$ no ha presentado esta dificultad; el 33,3\% dice haber presentado risa nerviosa, frente al 66,7\% que dice no haberlo hecho; otro tipo de conducta presentado en el $30,8 \%$ es aceptar haber tenido un trato brusco en sus relaciones sociales, pero el $69,2 \%$ no lo experimenta; se nota un $46,3 \%$ de personas que presentaron llanto mientras que el $53,7 \%$ dice que no ha presentado esta conducta; la característica de apretar las mandíbulas ha sido notada en el $26,0 \%$ de la muestra, frente al $74,0 \%$ que no lo demuestra; así mismo se informa por parte del 5,4\% haber tenido un aumento en el consumo de tabaco, y el 94,6\% no lo asegura; por último se reconoce por parte del 3,1\% haber ingerido alcohol mientras que el $96,9 \%$ no lo ha hecho.

En el criterio final sobre cambios físicos presentados, el 26,0\% dice haber notado sus músculos contraídos, el $74,0 \%$ no ha tenido esta afección; es importante el porcentaje del $68,4 \%$ de personas que indican haber tenido dolor de cabeza, en relación con el 31,6\% que no lo experimenta; así mismo con problemas de espalda o cuello un alto porcentaje del $70,6 \%$ frente al 
$29,4 \%$ que no muestra este cambio; el malestar estomacal se ha presentado en el $30,8 \%$ y no lo ha hecho en el $69,2 \%$ de la población analizada; La fatiga se ha presentado en el $47,5 \%$, frente al $52,5 \%$ que no lo ha tenido; Las infecciones se han notado en el $5,6 \%$ y en el $94,4 \%$ no han presentado este cambio; para terminar el estudio, afirman el $23,2 \%$ que han notado palpitaciones y respiración agitada mientras que el $76,8 \%$ no lo ha hecho.

Con el análisis de los resultados obtenidos, se confirma el incremento de todas las características e indicadores que permiten monitorear los niveles de estrés en los docentes y estudiantes universitarios que han sido afectados por el paso emergente de la educación presencial a la virtual en tiempos de pandemia y los venideros, lo cual vino para quedarse y es importante la adecuación a la nueva normalidad tratada por los medios y mucho más en un sistema de educación al cual nos enfrentamos.

Se indica en concreto, estar de acuerdo con la modalidad de educación online y la aplicación de herramientas tecnológicas por parte de las Instituciones de Educación Superior, además reconocen el haber postergado actividades importantes lo que provoca el incremento de los niveles de estrés manifestado en; aumento de ansiedad, irritabilidad, dificultad para concentrarse, preocupación sobre el futuro, dolor de cabeza, problemas de cuello y espalda, entre las características más presentadas de manera transversal.

Al describir los resultados más significativos de los niveles de estrés, producto de las conductas de procrastinación estudiadas, se propone un diagrama que concentra cinco fases que cumplen el ciclo Planificar, Hacer, Verificar y Actuar (PHVA), como se puede apreciar en la figura 1, de la mejora continua de Deming y que al ser practicadas, permitirán mitigar el riesgo al aumento del estrés mencionado por los docentes y estudiantes investigados y concienciará la importancia de reconocer el tema antes que se vuelva crónico, aportando de esta manera a mejorar la salud mental y física de quienes tengan 
acceso directo a la información. Todo lo mencionado pretende conseguir que los individuos puedan adecuar y utilizar sus tiempos con eficiencia y eficacia dentro de los procesos académicos y sociales que los vincula.

Figura 1. Propuesta de actividades que permitan minimizar el impacto del estrés producto de la procrastinación considerando el uso adecuado del tiempo.

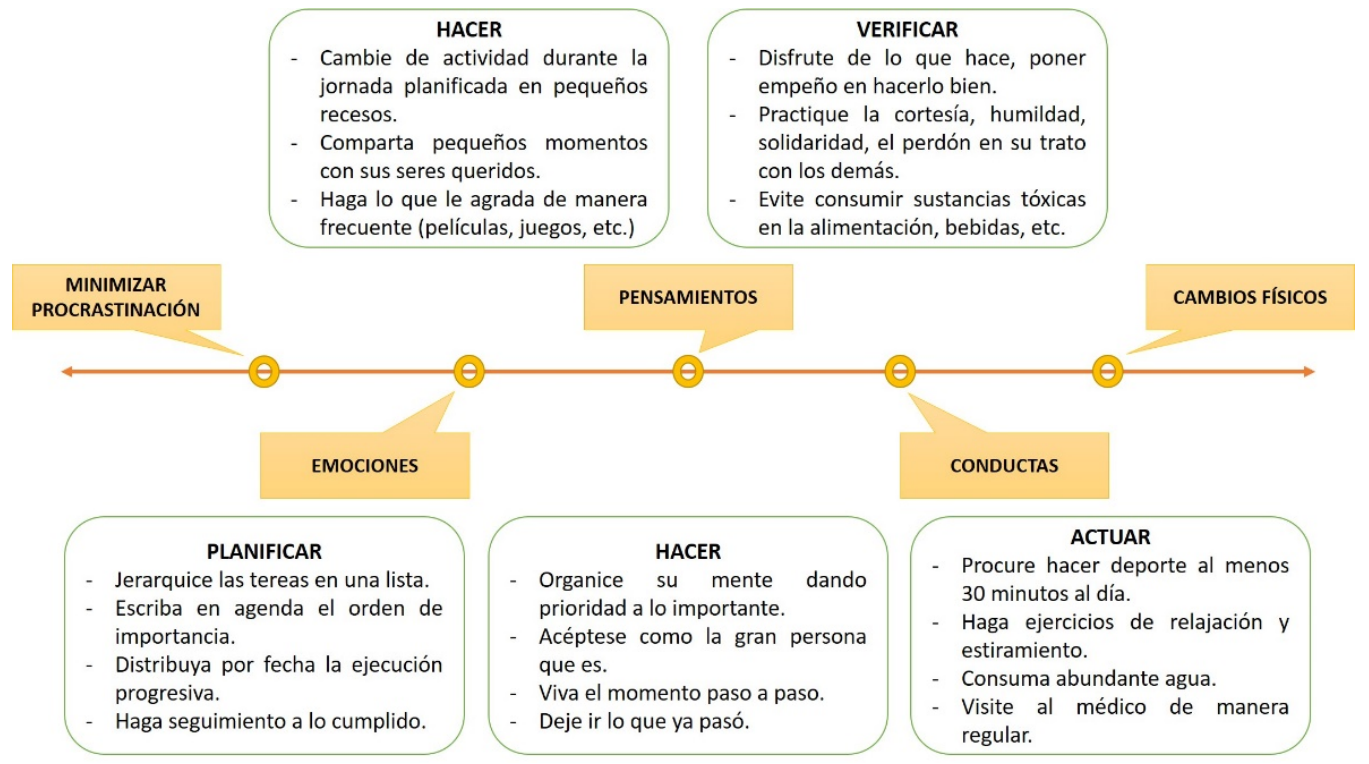

Fuente: El Autor (2021).

\section{Conclusiones}

La investigación en este estudio consiguió analizar el grado de incidencia de las conductas de procrastinación en los niveles de estrés en docentes y estudiantes universitarios frente al paso emergente a la educación online, se pudo verificar que muchas de las veces, dichas conductas se las pasan por alto y no se considera el riesgo provocado por la incidencia del estrés en el desarrollo de los procesos académicos notándose que no se han podido mitigar a tiempo.

Por lo tanto, se confirma el incremento de todas las características e indicadores que permiten monitorear los niveles de estrés en los docentes y 
estudiantes universitarios que han sido afectados por el paso emergente de la educación presencial a la virtual en tiempos de pandemia. Estos indicadores han priorizado el análisis de emociones, pensamiento, conductas e inclusive cambios físicos que se han visto afectados y que deben ser tratados adecuadamente en la nueva normalidad de un sistema de educación al cual nos enfrentamos y que vino para quedarse.

En cuanto a plantear alternativas de solución frente a la realidad encontrada, se propone dirigir estudios significativos hacia el uso adecuado del tiempo en todas las actividades académicas y sociales, con el fin de facilitar la consecución de las tareas cotidianas. La propuesta planteada en la presente investigación producto del análisis metodológico y de los resultados obtenidos, tiene como fin, facilitar la concreción y cumplimiento de las tareas académicas diarias, aportar con alternativas de solución minimizando el impacto en los detonantes del estrés y, desarrollar actitudes de apoyo a la gestión docente y discente de los universitarios motivo del estudio en mención.

\section{Referencias}

Atalaya, C., \& García, L. (2019a,b). Procrastinación: Revisión Teórica. Revista de Investigación en Psicología, 22(2), 363-378, e-ISSN: 16097475. Recuperado de: https://doi.org/10.15381/rinvp.v22i2.17435

Barba, M. (2019). Los menores marroquíes en Bizkaia, un colectivo encuarentena social. Inguruak, (67), 1-21, e-ISSN: 0214-7912. Recuperado de:

http://www.inguruak.eus/index.php/inguruak/article/view/150

Cueva, J., García, A., \& Martínez, O. (2019). El conectivismo y las TIC: Un paradigma que impacta el proceso enseñanza aprendizaje. Revista Scientific, 4(14), 205-227, e-ISSN: 2542-2987. Recuperado de: https://doi.org/10.29394/Scientific.issn.2542-2987.2019.4.14.10.205- 
Espinosa-Castro, F., Hernández-Lalinde, J., Rodríguez, J., Chacín, M., \& Bermúdez-Pirela, V. (2020). Influencia del estrés sobre el rendimiento académico. AVFT: Archivos Venezolanos de Farmacología y Terapéutica, 39(1), 63-69, e-ISSN: 2610-7988. Recuperado de:

http://www.revistaavft.com/images/revistas/2020/avft 1 2020/14 influ encia.pdf

Gené-Badia, J., Ruiz-Sánchez, M., Obiols-Masó, N., Oliveras, L., \& Lagarda, E. (2016). Aislamiento social y soledad: ¿qué podemos hacer los equipos de atención primaria?. Atención Primaria, 48(9), 604-609, eISSN: 0212-6567. Recuperado de:

https://doi.org/10.1016/j.aprim.2016.03.008

Moncada, J., \& Chacón, Y. (2012). El efecto de los videojuegos en variables sociales, psicológicas y fisiológicas en niños y adolescentes. Retos, 21, 43-49, e-ISSN: 1988-2041. Recuperado de:

https://doi.org/10.47197/retos.v0i21.34603

Nieto, R. (2012). Educación virtual o virtualidad de la educación. Revista Historia de la Educación Latinoamericana, 14(19), 137-150, e-ISSN: 0122-7238. Colombia: Universidad Pedagógica y Tecnológica de Colombia.

Silva-Ramos, M., López-Cocotle, J., \& Meza-Zamora, M. (2020). Estrés académico en estudiantes universitarios. Investigación y Ciencia de la Universidad Autónoma de Aguascalientes, (79), 75-83, e-ISSN: 25219758. Recuperado de: https://doi.org/10.33064/iycuaa2020792960

Suárez-Montes, N., \& Díaz-Subieta, L. (2015). Estrés académico, deserción y estrategias de retención de estudiantes en la educación superior. Revista de Salud Pública, 17(2), 300-313, ISSN: 0124-0064. Recuperado de: https://doi.org/10.15446/rsap.v17n2.52891 


\section{César Raúl Méndez Carpio \\ e-mail: $\underline{\text { cmendezc@ucacue.edu.ec }}$}

Nacido en Cuenca, Ecuador, el 22 de febrero del año 1971.

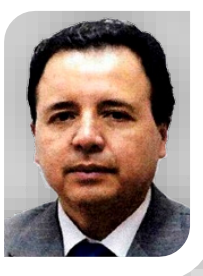

Dr. En Ciencias de la Educación, especialidad Ordenadores en la Universidad Católica de Cuenca (UCACUE); Magister en Docencia y Currículo para la Educación Superior en la Universidad Técnica de Ambato (UNITA); Magister en Administración de Empresas en la Universidad del Azuay (UDA); Actualmente me desempeño como Jefe de Investigación en el Colegio Militar "Abdón Calderón" y como docente en la Universidad Católica de Cuenca (UCACUE), en las Asignaturas: Estadística Aplicada, Metodología de Investigación, Tecnologías de Información y Comunicación.

El contenido de este manuscrito se difunde bajo una Licencia de Creative Commons ReconocimientoNoComercial-Compartirlgual 4.0 Internacional 